\title{
Appropriate indications for computed tomography coronary angiography
}

This clinical update draws on the recommendations of the Cardiac Society of Australia and New Zealand (CSANZ) 2010 guidelines on non-invasive coronary artery imaging, which we wrote on behalf of the Imaging Subcommittee of the CSANZ. The update provides additional pointers for medical practitioners who are considering computed tomography coronary angiography for their patients. The guidelines were independently reviewed by the Continuing Education and Recertification Committee, before being ratified by CSANZ Board in November 2010.

\section{Gary Y H Liew MB BS, FRACP Cardiologist-in-Charge, Cardiac CT Service}

Michael P Feneley MD, FRACP, Director of Cardiology

\section{Stephen G Worthley}

MD, PhD, FRACP Director of Catheterization Laboratories

1 Royal Adelaide Hospital, Adelaide, SA.

2St Vincent's Hospital, Sydney, NSW.

gary.liew@ adelaide.edu.au

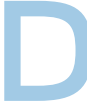
of this new technology in order to prevent its misuse.

\section{MDCT basics}

espite the various functional tests and biomarkers available for evaluation of patients with coronary artery disease (CAD), we sometimes look for the reassurance of anatomical information by way of a coronary angiogram. However, as an invasive modality, it does carry some risks, and the proportion of patients with normal coronary angiograms has remained relatively stable at $15 \% .{ }^{1}$

In recent years, the rapid development of computed tomography (CT) technology, reduction in radiation levels and introduction of a Medicare Benefits Schedule item number in 2011 have fuelled the growth of multidetector computed tomography (MDCT) around Australia as a noninvasive test for CAD. Therefore, it is important to have a good understanding of the appropriate use and limitations

The most common CT scanners used for cardiac imaging today have 64 detectors, arranged in the cranial-caudal direction, covering a distance of about $4 \mathrm{~cm}$ with each heartbeat and having a spatial resolution of $0.3-0.6 \mathrm{~mm}$. Respiratory motion is eliminated if the patient is able to hold his or her breath for about 10 seconds during the scan. Electrocardiogram (ECG) gating allows the scanner to obtain images during diastole when there is least motion of the coronaries. The computer then aligns the data from the different parts of the heart obtained during those five to

1 General recommendations for patients undergoing computed tomography coronary angiography (CTCA)*

- In sinus rhythm.

- Heart rate $<65$ beats per minute.

- Able to take $\beta$-blockers.

- Able to hold breath for 10 seconds.

Normal renal function (typical contrast volume used $<100 \mathrm{~mL}$ ).

- No previous contrast allergy.

Able to hold arms above head during scan.

- Most centres will administer sublingual glyceryl trinitrate for coronary vasodilatation.

* Not all are absolute requirements for CTCA; consult with individual diagnostic facility if patient does not fulfil some points.

\section{Summary}

- Computed tomography coronary angiography (CTCA) has been shown in multicentre trials to be reliable in ruling out significant coronary artery disease (CAD).

- It is used most appropriately in symptomatic patients with low to intermediate pretest probability of CAD.

- It should not be used in asymptomatic subjects, patients with known significant CAD or patients with a high pretest probability of CAD.

- The radiation dose of CTCA was previously two to three times that of invasive coronary angiography but with modern protocols, it is similar or lower.

- Patients generally need to be in sinus rhythm, tolerate $\beta$-blockers and nitrates, have a heart rate $<65$ beats per minute, be able to hold their breath for 10 seconds, and have normal renal function.

seven heartbeats to present a three-dimensional volumetric dataset. Multiplanar reconstructions of the images allow the reporter to cut through this 3-D dataset in any plane to demonstrate the coronaries in different axes. Abnormal movement, breathing, ectopy or arrhythmia during the scan will cause misalignment of the images, resulting in step artefacts, which may hamper interpretation. The general prerequisites for patients undergoing computed tomography coronary angiography (CTCA) in order to achieve optimum image quality are set out in Box 1 .

Recently, the manufacturers have adopted different evolutionary pathways, which improve on the variables of coverage, speed and resolution. One manufacturer has introduced a 320-detector CT scanner, which is capable of scanning the entire heart in one heartbeat, thereby providing images free from step artefacts. Another manufacturer has introduced its second-generation dual-source CT scanner, which has two sets of 128-detectors placed 90 degrees apart in the gantry so that it obtains images with only a quarter rotation. This has enabled good quality images at higher heart rates, as it can obtain images in half the time of other manufacturers' scanners. ${ }^{2}$ A third manufacturer has introduced a 64-detector CT scanner with improved resolution of $0.23 \mathrm{~mm}$, which enables better discrimination of fine objects like stents. ${ }^{3}$

\section{Radiation}

The amount of radiation delivered to the patient depends on a number of factors, such as patient size, sex, distance covered and scanning protocols. The traditional method of scanning is called retrospective scanning, where radiation is delivered throughout the cardiac cycle. ECG-gated dose 


\section{Average radiation dose, by procedure}

Procedure

Dose (mSv)

$\begin{array}{lc}\text { Annual background radiation (United States) } & 3 \\ \text { Chest x-ray (posteroanterior only) } & 0.02 \\ \text { Invasive coronary angiography } & 7 \\ \text { Calcium scoring on cardiac computed tomography } & 2 \\ \text { CTCA - prospective (120 kV) } & 2-5 \\ \text { CTCA - retrospective (120 kV) } & 12 \\ \text { Nuclear stress scan } & 11 \\ \text { Technetium-99m sestamibi } & 22 \\ \text { Thallium-201 } & \end{array}$

CTCA = computed tomography coronary angiography.

modulation is a setting that decreases radiation during systole, resulting in $25 \%-40 \%$ lower radiation for both men $(8 \mathrm{mSv})$ and women $(12 \mathrm{mSv})$.

In patients who weigh $<85 \mathrm{~kg}$ or have a body mass index $<30 \mathrm{~kg} / \mathrm{m}^{2}$, lowering the power setting of the scanner (from $120 \mathrm{kV}$ to $100 \mathrm{kV}$ ) reduces radiation by up to $60 \%$ while maintaining diagnostic quality. ${ }^{6}$ However, it has not been employed by some diagnostic facilities as they were unaware of this fact. ${ }^{4,6}$

A recent breakthrough is the prospective scanning technique, which delivers radiation only during a very short period in diastole. The radiation reduction is up to $80 \%$, with doses of $2-5 \mathrm{mSv}$, which is lower than typical invasive coronary angiograms and nuclear stress scans (Box 2). ${ }^{4,7}$ However, patients must have stable, low heart rates $(<60$ beats per minute) without ectopy or heart rate variability, as there is little margin for error. Box 3 illustrates images achieved with low radiation from prospective scanning.

\section{Diagnostic accuracy}

Meta-analyses of over 45 single-centre studies have consistently shown CTCA to have excellent sensitivity (98\%) and very good specificity ( $88 \%$ ) compared with invasive coronary angiography for significant disease (stenosis $>50 \%$ ). ${ }^{5,8,9}$ The negative predictive values $(96 \%-100 \%)$ were better than positive predictive values (93\%), demonstrating CTCA to be an excellent tool for ruling out significant disease in patients with low-to-intermediate pretest probability of CAD. Similar results were found in prospective multicentre and multivendor validation trials of CTCA. ${ }^{10-12}$

The prognostic value of non-obstructive disease on CTCA has been investigated. One study involving 1256 patients with up to 2 years of follow-up found that, of 802 patients with mild disease on CTCA, only one patient $(0.12 \%)$ had a severe cardiac event in the form of unstable angina. ${ }^{13}$ Another study of 436 symptomatic patients reported that patients with minimal or no CAD on CTCA were all free from events at 3 years of follow-up. ${ }^{14}$

Although CTCA can reliably exclude obstructive disease based on excellent negative predictive values, its ability to quantify stenosis severity is not as robust. Studies comparing CTCA to quantitative coronary angiography and intravascular ultrasound found good correlations but large standard deviations (up to $\pm 25 \%$ ). ${ }^{10,15}$ Therefore, the Society of Cardiovascular Computed Tomography has recommended that stenoses be graded in broad ranges rather than

\section{Prospective computed tomography scan images}

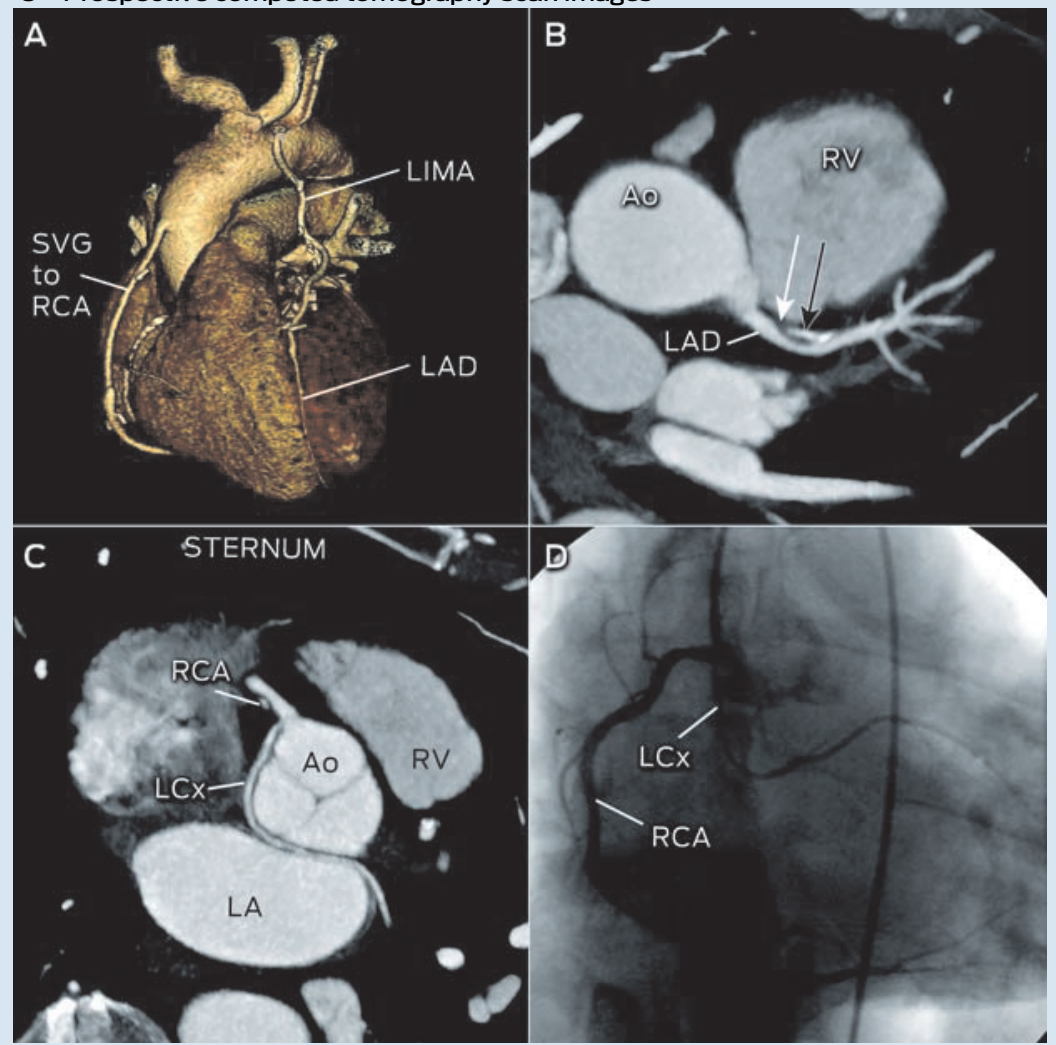

A: Volume-rendered tomogram of Patient 1, showing location of previous bypass grafts (radiation $=4.2 \mathrm{mSv} ; 120 \mathrm{kV}$ ). B: Computed tomography coronary angiogram of Patient 2 with chest pain, showing moderate $L A D$ stenosis caused by mixed plaque consisting of non-calcified (white arrow) and calcified (black arrow) plaque (radiation $=1.1 \mathrm{mSv}$; $100 \mathrm{kV}$, no padding). C: Patient 2, demonstrating anomalous $L C X$ originating from RCA that has mild mixed plaque. LCX takes a "non-malignant" route between the aorta and left atrium, which is unlikely to experience compression. $D$ : Corresponding invasive angiogram of Patient 2, demonstrating anomalous $L C x$. Ao = aorta. $L A=$ left atrium. $L A D=$ left anterior descending artery. $L C X=$ left circumflex artery. $L I M A=$ left internal mammary artery. $R C A=$ right coronary artery. $R V=$ right ventricle. $S V G=$ saphenous vein graft. Images from Cardiac CT Service, Royal Adelaide Hospital, using a 128-detector computed tomography scanner (SOMATOM Definition AS+, Siemens, Erlangen, Germany).

assigning specific numbers in their guidelines (Box 4). ${ }^{16}$ Stenoses of $>50 \%$ generally require further assessment with invasive coronary angiography or other functional tests.

\section{Appropriate indications for CTCA}

In 2011, the CSANZ published comprehensive guidelines on non-invasive coronary artery imaging. ${ }^{17}$ These are similar to those of the American multisociety ${ }^{18}$ and European Society of Cardiology ${ }^{19}$ guidelines. The appropriate indications for performing CTCA are outlined in

\begin{tabular}{|c|c|c|}
\hline Grade & Range & Recommendation \\
\hline Normal & No stenosis or plaque* & Medical management \\
\hline Minimal & $<25 \%$ stenosis & Medical management \\
\hline Mild & $25 \%-49 \%$ & Medical management \\
\hline Moderate & $50 \%-69 \%$ & Further assessment \\
\hline Severe & $\geqslant 70 \%$ & Further assessment \\
\hline
\end{tabular}


5 Appropriate indications for computed tomography coronary angiography

- Evaluation of chest pain with no previous known disease:

able to exercise and no previous tests (intermediate risk)

unable to exercise or ECG uninterpretable (low-to-intermediate risk)

equivocal or uninterpretable stress test results

normal ECG exercise test but ongoing symptoms.

- Evaluation of acute chest pain (emergency department):

normal ECG and cardiac enzymes

low-to-intermediate pretest probability of CAD.

- Evaluation of suspected coronary anomalies/complex congenital heart disease.

Exclusion of CAD in new-onset heart failure/cardiomyopathy.

- Assessment of CABG patency and vascular mapping before repeat CABG surgery.

- Exclusion of significant CAD before non-coronary cardiac surgery.

Investigation of left bundle branch block for suspected CAD as an aetiology.

$\mathrm{CABG}=$ coronary artery bypass graft. $\mathrm{CAD}=$ coronary artery disease. $\mathrm{ECG}=$ electrocardiogram.

Box 5. The discussion below includes some of the more common scenarios.

\section{Evaluation of stable chest pain}

The diagnostic accuracy of CTCA in the assessment of stable chest pain in low-to-intermediate-risk patients is discussed above. In terms of cost-effectiveness, it is uncertain which position CTCA should occupy in the stable chest pain diagnostic pathway. Complex computer simulation models of using CTCA before, after and instead of various stress test modalities have shown it to be comparable to those stress and functional tests already available. ${ }^{20}$ Large randomised controlled trials are currently underway examining CTCA versus various stress-testing modalities as the initial strategy for chest pain.

\section{Evaluation of acute chest pain (emergency department)}

There have been a few small single-centre trials in the United States assessing the use of CTCA in the setting of acute chest pain..$^{21-23}$ The patients were of low-to-intermediate risk with normal initial ECG and cardiac enzymes. The studies showed that if there was no obstructive disease on CTCA, the patients were safe for early discharge without serious cardiac events in the follow-up period. One study showed time and cost savings due to early triage and management of patients. ${ }^{22}$ However, further studies into local cost-effectiveness and workflow pathways need to be conducted before routine use in Australia and New Zealand can be recommended. ${ }^{17}$

\section{Evaluation of new-onset heart failure or cardiomyopathy}

In the evaluation of patients with new-onset heart failure or dilated cardiomyopathy, invasive coronary angiography is often performed to rule out CAD as a contributing factor. In a study comparing CTCA with invasive angiography where $32 \%$ of patients had significant lesions, CTCA had excellent accuracy (>99\%) for detecting stenoses of $>50 \%$ and $>70 \%$. $^{24}$

\section{Investigation of left bundle branch block}

Left bundle branch block carries an increased risk of cardiac events and can be associated with CAD. The detection of significant lesions often requires invasive coronary angiography because stress testing and imaging
6 Questions to ask diagnostic facilities performing computed tomography coronary angiography

- Is the reporting doctor qualified to at least CCRT-CTCA Level A?

- Do the reports state radiation dose (mSv) and scanning technique?

- Does the facility routinely use lower power $(100 \mathrm{kV})$ for patients who weigh $<85 \mathrm{~kg}$ ?

Is prospective scanning available?

CCRT-CTCA = Australia and New Zealand Conjoint Committee for Recognition of Training in computed tomography coronary angiography.

can be unreliable in the presence of left bundle branch block. CTCA demonstrated high accuracy (95\%) with an excellent negative predictive value (97\%) compared with invasive angiography in a patient cohort with a $44 \%$ prevalence of significant CAD. ${ }^{25}$

\section{Evaluation of coronary stents}

When a patient with previous stents presents with stable symptoms, a major concern is in-stent restenosis. This can be challenging for CTCA because there are various artefacts created by the stents, which may impede accurate assessment. Stent diameter $<3 \mathrm{~mm}$ has been identified as a major predictor of an unevaluable stent. ${ }^{26}$ Therefore, routine use of CTCA to evaluate stent patency is not recommended, except in very selective cases of large stents and simple left main stents. ${ }^{17}$

\section{Risk stratification of asymptomatic patients}

The current data do not support the use of CTCA to detect CAD in asymptomatic individuals. ${ }^{27}$ However, there is evidence for coronary artery calcium (CAC) scoring using non-contrast cardiac CT scans in asymptomatic intermediate-risk individuals. ${ }^{28}$ It has been shown to provide independent and incremental prognostic information over Framingham risk score alone. ${ }^{29}$ The reclassification to a different risk group by CAC score influenced eligibility for statin therapy when applying guidelines on heart attack prevention. ${ }^{30}$ Furthermore, a CAC score of zero confers a very low cardiac event rate of $<0.1 \%$ per year. ${ }^{31}$

\section{Conclusion}

It is now possible to exclude severe coronary artery stenosis non-invasively by CTCA. Current evidence supports its use in symptomatic individuals with select indications. There exists the potential for misuse with this emerging modality, and consideration should be given to other options in light of local resources and expertise (Box 6). The rapid development in technology and further research will clarify and expand the role of cardiac CT in the future.

\section{Competing interests: No relevant disclosures.}

Provenance: Not commissioned; peer reviewed.

1 Farrehi PM, Bernstein SJ, Rasak M, et al. Frequency of negative coronary arteriographic findings in patients with chest pain is related to community practice patterns. Am J Manag Care 2002; 8: 643-648.

2 Ropers U, Ropers D, Pflederer T, et al. Influence of heart rate on the diagnostic accuracy of dual-source computed tomography coronary angiography. J Am Coll Cardiol 2007; 50: 2393-2398.

3 Min JK, Swaminathan RV, Vass M, et al. High-definition multidetector computed tomography for evaluation of coronary artery stents: comparison to standard-definition 64-detector row computed tomography. J Cardiovasc Comput Tomogr 2009; 3: 246-251. 
4 Hausleiter J, Meyer T, Hermann F, et al. Estimated radiation dose associated with cardiac CT angiography. JAMA 2009; 301: 500-507.

5 Mowatt G, Cook JA, Hillis GS, et al. 64-Slice computed tomography angiography in the diagnosis and assessment of coronary artery disease: systematic review and meta-analysis. Heart 2008; 94: 1386-1393.

6 Raff GL, Chinnaiyan KM, Share DA, et al. Radiation dose from cardiac computed tomography before and after implementation of radiation dose-reduction techniques. JAMA 2009; 301: 2340-2348.

7 Einstein AJ, Moser KW, Thompson RC, et al. Radiation dose to patients from cardiac diagnostic imaging. Circulation 2007; 116: 1290-1305.

8 Abdulla J, Abildstrom SZ, Gotzsche 0, et al. 64-multislice detector computed tomography coronary angiography as potential alternative to conventional coronary angiography: a systematic review and meta-analysis. Eur Heart 12007 ; 28: 3042-3050.

9 Stein PD, Yaekoub AY, Matta F, et al. 64-slice CT for diagnosis of coronary artery disease: a systematic review. Am J Med 2008; 121: 715-725.

10 Miller JM, Rochitte CE, Dewey M, et al. Diagnostic performance of coronary angiography by 64-row CT. N Engl J Med 2008; 359: 2324-2336

11 Meijboom WB, Meijs MF, Schuijf JD, et al. Diagnostic accuracy of 64-slice computed tomography coronary angiography: a prospective, multicenter, multivendor study. J Am Coll Cardiol 2008; 52: 2135-2144.

12 Budoff MJ, Dowe D, Jollis JG, et al. Diagnostic performance of 64-multidetector row coronary computed tomographic angiography for evaluation of coronary artery stenosis in individuals without known coronary artery disease: results from the prospective multicenter ACCURACY (Assessment by Coronary Computed Tomographic Angiography of Individuals Undergoing Invasive Coronary Angiography) trial. J Am Coll Cardiol 2008; 52: 1724-1732.

13 Hadamitzky M, Freissmuth B, Meyer T, et al. Prognostic value of coronary computed tomographic angiography for prediction of cardiac events in patients with suspected coronary artery disease. JACC Cardiovasc Imaging 2009; 2: 404-411.

14 Fazel P, Peterman MA, Schussler JM. Three-year outcomes and cost analysis in patients receiving 64-slice computed tomographic coronary angiography for chest pain. Am J Cardiol 2009; 104: 498-500.

15 Leber AW, Knez A, von Ziegler F, et al. Quantification of obstructive and nonobstructive coronary lesions by 64-slice computed tomography: a comparative study with quantitative coronary angiography and intravascular ultrasound. J Am Coll Cardiol 2005; 46: 147-154.

16 Raff GL, Abidov A, Achenbach S, et al. SCCT guidelines for the interpretation and reporting of coronary computed tomographic angiography. J Cardiovasc Comput Tomogr 2009; 3: 122-136.

17 Liew GY, Feneley M, Worthley SG. Noninvasive coronary artery imaging: current clinical applications: Cardiac Society of Australia and New Zealand guidelines. Heart Lung Circ 2011; 20: 425-437.

18 Taylor AJ, Cerqueira M, Hodgson JM, et al. ACCF/SCCT/ACR/AHA/ASE/ASNC/ NASCI/SCAI/SCMR 2010 Appropriate Use Criteria for Cardiac Computed Tomography: A Report of the American College of Cardiology Foundation Appropriate Use Criteria Task Force, the Society of Cardiovascular Computed Tomography, the American College of Radiology, the American Heart Association, the American Society of Echocardiography, the American Society of Nuclear Cardiology, the North American Society for Cardiovascular Imaging, the Society for Cardiovascular Angiography and Interventions, and the Society for Cardiovascular Magnetic Resonance. Circulation 2010; 122: e525-e555.

19 Schroeder S, Achenbach S, Bengel F, et al. Cardiac computed tomography: indications, applications, limitations, and training requirements: report of a Writing Group deployed by the Working Group Nuclear Cardiology and Cardiac CT of the European Society of Cardiology and the European Council of Nuclear Cardiology. Eur Heart J 2008; 29: 531-556.

20 Ladapo JA, Jaffer FA, Hoffmann U, et al. Clinical outcomes and costeffectiveness of coronary computed tomography angiography in the evaluation of patients with chest pain. J Am Coll Cardiol 2009; 54: 2409-2422.

21 Hoffmann U, Bamberg F, Chae CU, et al. Coronary computed tomography angiography for early triage of patients with acute chest pain: the ROMICAT (Rule Out Myocardial Infarction using Computer Assisted Tomography) trial. J Am Coll Cardiol 2009; 53: 1642-1650.

22 Goldstein JA, Gallagher MJ, O'Neill WW, et al. A randomized controlled trial of multi-slice coronary computed tomography for evaluation of acute chest pain. J Am Coll Cardiol 2007; 49: 863-871.

23 Rubinshtein R, Halon DA, Gaspar T, et al. Usefulness of 64-slice cardiac computed tomographic angiography for diagnosing acute coronary syndromes and predicting clinical outcome in emergency department patients with chest pain of uncertain origin. Circulation 2007; 115: 1762-1768.

24 Andreini D, Pontone G, Bartorelli AL, et al. Sixty-four-slice multidetector computed tomography: an accurate imaging modality for the evaluation of coronary arteries in dilated cardiomyopathy of unknown etiology. Circ Cardiovasc Imaging 2009; 2: 199-205.

25 Ghostine S, Caussin C, Daoud B, et al. Non-invasive detection of coronary artery disease in patients with left bundle branch block using 64-slice computed tomography. J Am Coll Cardiol 2006; 48: 1929-1934.

26 Rixe J, Achenbach S, Ropers D, et al. Assessment of coronary artery stent restenosis by 64-slice multi-detector computed tomography. Eur Heart $\mathrm{J} 2006$; 27: 2567-2572.

27 Choi EK, Choi SI, Rivera JJ, et al. Coronary computed tomography angiography as a screening tool for the detection of occult coronary artery disease in asymptomatic individuals. J Am Coll Cardiol 2008; 52: 357-365.

28 Greenland P, Alpert JS, Beller GA, et al. 2010 ACCF/AHA guideline for assessment of cardiovascular risk in asymptomatic adults: executive summary: a report of the American College of Cardiology Foundation/American Heart Association Task Force on Practice Guidelines. Circulation 2010; 122: 2748-2764.

29 Greenland P, LaBree L, Azen SP, et al. Coronary artery calcium score combined with Framingham score for risk prediction in asymptomatic individuals. JAMA 2004; 291: 210-215

30 See R, Lindsey JB, Patel MJ, et al. Application of the screening for Heart Attack Prevention and Education Task Force recommendations to an urban population: observations from the Dallas Heart Study. Arch Intern Med 2008; 168: 1055-1062.

31 Arad Y, Goodman KJ, Roth M, et al. Coronary calcification, coronary disease risk factors, C-reactive protein, and atherosclerotic cardiovascular disease events: the St. Francis Heart Study. J Am Coll Cardiol 2005; 46: 158-165.

\section{Raffaela Armiento Intern, Department of Medicine \\ llana Gory Gastroenterology Registrar \\ Peter J Jenkins Gastroenterologist \\ Catriona A McLean Anatomical Pathologist Alfred Health, Melbourne, VIC. \\ raff.armiento@ gmail.com \\ Calciphylaxis and hypoalbuminaemia \\ A 58-year-old woman presented with multiple extensive, necrotic ulcers on the proximal lower limbs (Figure, A and B). A diagnosis of calciphylaxis in the absence of renal impairment was made. Although non-uraemic calciphylaxis has been described previously, the pathogenic mechanisms and significance of documented risk factors are poorly understood. Multiple risk factors were identified in this patient, of which the most prominent is likely to have been severe and longstanding \\ hypoalbuminaemia of unexplained cause. In addition, obesity, European ancestry, female sex, hypovitaminosis $\mathrm{D}$, an elevated alkaline phosphatase level, non-alcoholic steatohepatitis, weight loss and use of steroids were all features of this case that have documented associations with calciphylaxis. \\ Acknowledgements: We thank the staff of the Departments of General Medicine, Dermatology and Anatomical Pathology at the Alfred Hospital for their contribution. doi: 10.5694/mjall.10894

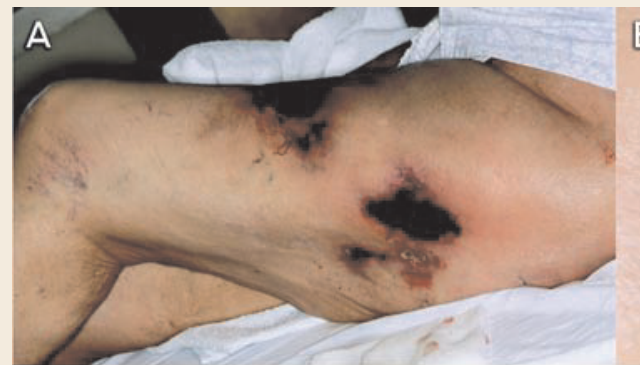

A: Extensive ulceration of the left lateral thigh.

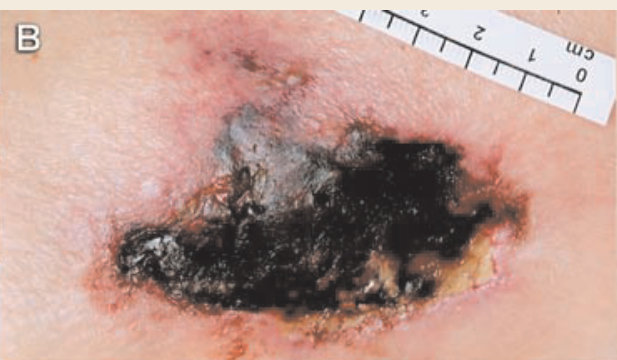

B: Extensive ulceration of the left lateral thigh.

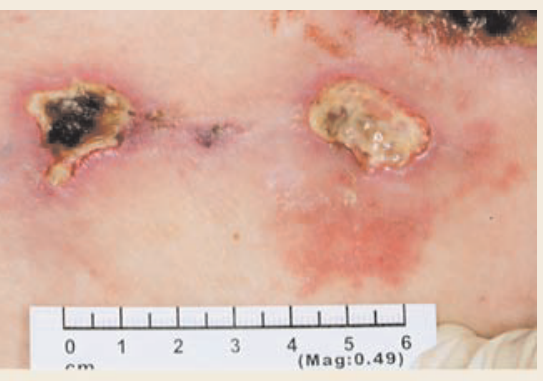

$\sim m$ 